\title{
Dystrophic epidermolysis bullosa pruriginosa
}

INSERM

\section{Source}

INSERM. (1999). Orphanet: an online rare disease and orphan drug data base. Dystrophic epidermolysis bullosa pruriginosa. ORPHA:89843

Dystrophic epidermolysis bullosa pruriginosa is a rare subtype of dystrophic epidermolysis bullosa (DEB, see this term) characterized by generalized or localized skin lesions associated with severe, if not intractable, pruritus. 